University of Nebraska - Lincoln

DigitalCommons@University of Nebraska - Lincoln

\title{
Separation and identification of soybean leaf proteins by two- dimensional gel electrophoresis and mass spectrometry
}

\author{
Chenping $\mathrm{Xu}$ \\ University of Maryland - College Park \\ Wesley M. Garrett \\ USDA-ARS, wesley.garrett@ars.usda.gov \\ Joseph Sullivan \\ University of Maryland - College Park \\ Thomas J. Caperna \\ United States Department of Agriculture-Agricultural Research Service \\ Savithiry Natarajan \\ United States Department of Agriculture-Agricultural Research Service, natarajs@ba.ars.usda.gov
}

Follow this and additional works at: https://digitalcommons.unl.edu/usdaarsfacpub

Part of the Agricultural Science Commons

Xu, Chenping; Garrett, Wesley M.; Sullivan, Joseph; Caperna, Thomas J.; and Natarajan, Savithiry, "Separation and identification of soybean leaf proteins by two-dimensional gel electrophoresis and mass spectrometry" (2006). Publications from USDA-ARS / UNL Faculty. 695.

https://digitalcommons.unl.edu/usdaarsfacpub/695

This Article is brought to you for free and open access by the U.S. Department of Agriculture: Agricultural Research Service, Lincoln, Nebraska at DigitalCommons@University of Nebraska - Lincoln. It has been accepted for inclusion in Publications from USDA-ARS / UNL Faculty by an authorized administrator of DigitalCommons@University of Nebraska - Lincoln. 


\title{
Separation and identification of soybean leaf proteins by two-dimensional gel electrophoresis and mass spectrometry is
}

\author{
Chenping $\mathrm{Xu}{ }^{\text {a }}$, Wesley M. Garrett ${ }^{\mathrm{b}}$, Joseph Sullivan ${ }^{\mathrm{a}}$, \\ Thomas J. Caperna ${ }^{c}$, Savithiry Natarajan ${ }^{\mathrm{d}, *}$ \\ a Department of Natural Resource Sciences and Landscape Architecture, University of Maryland, College Park, MD 20742, USA \\ ${ }^{\mathrm{b}}$ US Department of Agriculture-Agricultural Research Service, Biotechnology and Germplasm Laboratory, Beltsville, MD 20705, USA \\ ${ }^{\mathrm{c}}$ US Department of Agriculture-Agricultural Research Service, Growth Biology Laboratory, Beltsville, MD 20705, USA \\ ${ }^{\mathrm{d}}$ US Department of Agriculture-Agricultural Research Service, Soybean Genomics and Improvement Laboratory, PSI, Beltsville, MD 20705, USA
}

Received 23 December 2005; received in revised form 17 February 2006

\begin{abstract}
To establish a proteomic reference map for soybean leaves, we separated and identified leaf proteins using two-dimensional polyacrylamide gel electrophoresis (2D-PAGE) and mass spectrometry (MS). Tryptic digests of 260 spots were subjected to peptide mass fingerprinting (PMF) by matrix-assisted laser desorption/ionization-time of flight (MALDI-TOF) MS. Fifty-three of these protein spots were identified by searching NCBInr and SwissProt databases using the Mascot search engine. Sixty-seven spots that were not identified by MALDI-TOF-MS analysis were analyzed with liquid chromatography tandem mass spectrometry (LC-MS/MS), and 66 of these spots were identified by searching against the NCBInr, SwissProt and expressed sequence tag (EST) databases. We have identified a total of 71 unique proteins. The majority of the identified leaf proteins are involved in energy metabolism. The results indicate that 2D-PAGE, combined with MALDI-TOF-MS and LC-MS/MS, is a sensitive and powerful technique for separation and identification of soybean leaf proteins. A summary of the identified proteins and their putative functions is discussed.
\end{abstract}

(c) 2006 Elsevier Ltd. All rights reserved.

Keywords: Glycine max; Soybean; Proteomics; Two-dimensional gel electrophoresis; MALDI-TOF MS; LC-MS/MS

\section{Introduction}

The two key steps in classical proteomics are the separation of proteins and their subsequent identification. In a standard approach, two-dimensional polyacrylamide gel

\footnotetext{
Mention of trade name, proprietary product or vendor does not constitute a guarantee or warranty of the product by the US Department of Agriculture or imply its approval to the exclusion of other products or vendors that also may be suitable.

* Corresponding author. Address: PSI-ARS-USDA, Soybean Genomics and Improvement Laboratory, 10300, Baltimore Avenue, Beltsville, MD 20705, USA. Tel.: +1 301504 5258; fax: +1 3015045728.

E-mail address: natarajs@ba.ars.usda.gov (S. Natarajan).
}

electrophoresis (2D-PAGE) and mass spectrometry (MS) are combined. Two-dimensional PAGE, in which proteins are separated according to their isoelectric point (pI) in the first dimension and molecular weight $(\mathrm{Mr})$ in the second dimension, is still the preferred separation technique of many researchers in the global and comparative analysis of proteins. MS has essentially replaced the classical technique of Edman degradation in protein identification because it is more sensitive, can deal with protein mixtures, and offers much higher throughput. There are two main approaches to MS protein identification. In peptide mass fingerprinting (PMF), the unknown protein is digested with a protease of known specificity such as trypsin. By determining the masses of the resulting peptides, a mass map 
or mass fingerprint can be obtained. This mass map is then compared with predicted mass maps of proteins within the database. The tandem mass spectrometric (MS/MS) method relies on fragmentation of individual peptides to obtain sequence information.

A number of plant proteomic studies have recently been published. Some focused on organelle or subcellular proteomes such as the chloroplast (Ferro et al., 2003; Lonosky et al., 2004), the mitochondria (Kruft et al., 2001; Bardel et al., 2002) or the ribosome (Yamaguchi et al., 2000, 2002), whereas others have focused on a specific tissue, such as Arabidopsis seeds (Gallardo et al., 2001), maize root (Chang et al., 2000) and maize leaves (Porubleva et al., 2001), soybean seed (Mooney and Thelen, 2004; Natarajan et al., 2005), pea leaves (Schiltz et al., 2004) and legume barrel medic roots (Mathesius et al., 2001). Large-scale projects to identify proteins from multiple tissues of the barrel medic (Watson et al., 2003), rice (Komatsu et al., 2004) and Arabidopsis (Giavalisco et al., 2005) have also been reported.

Soybean, Glycine max (G. max), provides an inexpensive source of protein for human food and for the animal industry and has been the dominant oilseed produced since the 1960s. So far, no protein reference map has been reported for soybean leaves. As a first step to study stress physiology of soybean, we separated and identified soybean leaf proteins from normal plants. We describe here the extraction and separation of soybean leaf proteins on 2D-PAGE gels and identification of proteins using both MALDI-TOFMS and LC-MS/MS. The Mascot search engine was used to search against NCBInr, SwissProt and EST databases for protein identification.

\section{Results and discussion}

\subsection{Separation of soybean leaf proteins}

A previously described protein extraction protocol using acetone/trichloroacetic acid (TCA) precipitation was employed for the extraction of protein from soybean leaf (Natarajan et al., 2005). The proteins separated by 2DPAGE were visualized by Coomassie brilliant blue (CBB) G-250 staining, which is MS compatible and allows reproducible protein detection. The resulting 2D-PAGE images, which were reproduced from four independent biological experiments, constitute the reference gel images. The simplicity of the protocol favors the reproducibility of the protein separation. A representative 2D-PAGE protein pattern of soybean leaf is presented in Fig. 1. The dynamic range of protein accumulation is very large; this is a problem for leaf proteomic analysis because the preponderance of ribulose bisphosphate carboxylase/oxygenase (Rubisco) masks the detection of other proteins (Wilson et al., 2002; Watson et al., 2003). However, in our system a significant number of proteins were clearly separated and identified despite the predominance of Rubisco in the central portion of the gels.

\subsection{Identification of separated proteins}

To qualitatively survey the proteins visualized by $2 \mathrm{D}$ PAGE, a total of 260 protein spots were excised from the 2D-PAGE gels and digested with the trypsin. The peptide fragments were extracted and analyzed by MALDI-TOFMS. Typically, high-quality MALDI-TOF-MS peptide

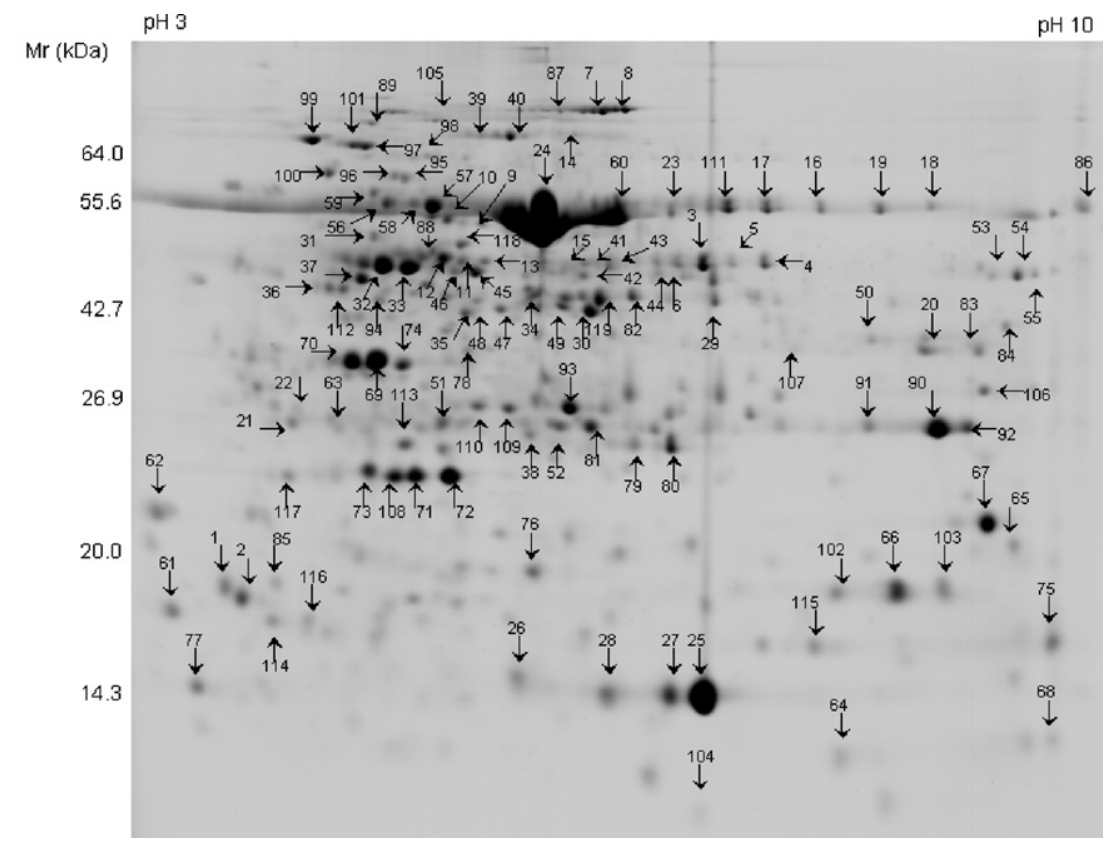

Fig. 1. Coomassie stained 2D-PAGE gel of separated soybean leaf proteins. Proteins were separated in the first dimension on a IPG strip pH 3.0-10.0 and in the second dimension on a $12.5 \%$ acrylamide SDS-gel. The numbered spots were identified and the derived data are presented in Table 1. 
Table 1

Proteins identified from the soybean leaf by MALDI-TOF-MS and LC-MS/MS

\begin{tabular}{|c|c|c|c|c|c|c|c|c|}
\hline SID & Protein identification [species] & T. Mr/pI & MO & PM & $\mathrm{SC}(\%)$ & Acce. no. & Databases & ID Method \\
\hline \multicolumn{9}{|c|}{ Functional category 1: Metabolism } \\
\hline 1 & H-protein of glycine cleavage system [ Glycine soja $]$ & $17744 / 4.59$ & 65 & 2 & 10 & gi|26045029 & EST_others & LC-MS/MS \\
\hline 2 & H-protein of glycine cleavage system [ Glycine soja $]$ & $17744 / 4.59$ & 145 & 3 & 22 & gi|26045029 & EST_others & LC-MS/MS \\
\hline 3 & T-protein of the glycine cleavage system [Pisum sativum $]$ & $44661 / 8.79$ & 131 & 3 & 7 & gi|407475 & NCBInr & LC-MS/MS \\
\hline 4 & T-protein of the glycine cleavage system [Pisum sativum] & $44661 / 8.79$ & 506 & 10 & 25 & gi|407475 & NCBInr & LC-MS/MS \\
\hline 5 & $\begin{array}{l}\text { T-protein of glycine cleavage system, mitochondrial } \\
\text { precursor }[\text { Pisum sativum }]\end{array}$ & $44656 / 8.79$ & 65 & 8 & 25 & P49364 & SwissProt & MALDI-TOF \\
\hline 6 & $\begin{array}{l}\text { T-protein of glycine cleavage system, mitochondrial } \\
\text { precursor [Pisum sativum] }\end{array}$ & $44661 / 8.79$ & 151 & 3 & 7 & gi|407475 & NCBInr & LC-MS/MS \\
\hline 7 & P-protein of glycine cleavage system, [Arabidopsis thaliana] & $114672 / 6.18$ & 463 & 9 & 10 & gi|3413705 & NCBInr & LC-MS/MS \\
\hline 8 & P-protein of glycine cleavage system, [Arabidopsis thaliana] & $114672 / 6.18$ & 514 & 12 & 12 & gi|3413705 & NCBInr & LC-MS/MS \\
\hline 9 & Alanine aminotransferase $[$ Arabidopsis thaliana $]$ & $53780 / 6.49$ & 164 & 4 & 9 & gi|23297208 & NCBInr & LC-MS/MS \\
\hline 10 & Alanine aminotransferase [Arabidopsis thaliana] & $53780 / 6.49$ & 128 & 3 & 7 & gi|23297208 & NCBInr & LC-MS/MS \\
\hline 11 & Glutamine synthetase precursor [Glycine $\max ]$ & $47948 / 6.73$ & 83 & 12 & 35 & gi|13877511 & NCBInr & MALDI-TOF \\
\hline 12 & Glutamine synthetase precursor [Glycine max] & $47948 / 6.73$ & 70 & 9 & 21 & gi|13877511 & NCBInr & MALDI-TOF \\
\hline 13 & Glutamine synthetase precursor [Glycine max] & $47948 / 6.73$ & 407 & 12 & 14 & gi|13877511 & NCBInr & LC-MS/MS \\
\hline 14 & Methionine synthase [Glycine $\max ]$ & $84401 / 5.93$ & 143 & 24 & 36 & gi|33325957 & NCBInr & MALDI-TOF \\
\hline 15 & Aspartate transaminase AAT5 precursor [Glycine max $]$ & $50725 / 7.16$ & 81 & 14 & 36 & gi|485495 & NCBInr & MALDI-TOF \\
\hline 16 & Glycine hydroxymethyltransferase [Flaveria pringlei] & $57127 / 8.72$ & 71 & 17 & 30 & gi|437995 & NCBInr & MALDI-TOF \\
\hline 17 & Glycine hydroxymethyltransferase [Flaveria pringlei] & $57068 / 8.8$ & 234 & 4 & 10 & gi|437997 & NCBInr & LC-MS/MS \\
\hline 18 & $\begin{array}{l}\text { Glycine hydroxymethyltransferase, mitochondrial } \\
\text { precursor[Solanum tuberosum] }\end{array}$ & $57224 / 8.40$ & 55 & 19 & 29 & P50433 & SwissProt & MALDI-TOF \\
\hline 19 & $\begin{array}{l}\text { Glycine hydroxymethyltransferase, mitochondrial } \\
\text { precursor [Arabidopsis thaliana] }\end{array}$ & $57534 / 8.4$ & 157 & 2 & 5 & Q9SZJ5 & SwissProt & LC-MS/MS \\
\hline 20 & Probable $\gamma$-glutamyl hydrolase [Glycine max] & $37824 / 6.08$ & 92 & 9 & 29 & gi|7488702 & NCBInr & MALDI-TOF \\
\hline 21 & Ribose 5-phosphate isomerase [Glycine max] & $19726 / 4.69$ & 386 & 6 & 40 & gi| 15285625 & EST_others & LC-MS/MS \\
\hline 22 & Ribose 5-phosphate isomerase [Glycine max] & $19726 / 4.69$ & 118 & 2 & 15 & gi|15285625 & EST_others & LC-MS/MS \\
\hline \multicolumn{9}{|c|}{ Functional category 2: Energy/pentose phosphate } \\
\hline 23 & Rubisco large subunit [Ophioglossum engelmannii] & $53034 / 5.96$ & 104 & 15 & 33 & gi|309636 & NCBInr & MALDI-TOF \\
\hline 24 & Rubisco large subunit [Glycine max] & $52802 / 6.09$ & 163 & 16 & 33 & gi|3114769 & NCBInr & MALDI-TOF \\
\hline 6 & $\begin{array}{l}\text { Ribulose-1,5-bisphosphate carboxylase [Lechenaultia } \\
\text { heteromera] }\end{array}$ & $43938 / 6.36$ & 152 & 4 & 10 & gi 1304320 & NCBInr & LC-MS/MS \\
\hline 25 & Rubisco small subunit rbcS2 [Glycine max $]$ & $20220 / 8.87$ & 150 & 14 & 57 & gi|10946377 & NCBInr & MALDI-TOF \\
\hline 26 & Rubisco small subunit rbcS2 [Glycine max] & $20220 / 8.87$ & 91 & 6 & 28 & gi|10946377 & NCBInr & MALDI-TOF \\
\hline 27 & Rubisco small subunit rbcS2 [Glycine max] & $20220 / 8.87$ & 175 & 15 & 57 & gi|10946377 & NCBInr & MALDI-TOF \\
\hline 28 & Rubisco small chain 4 , chloroplast precursor [Glycine max] & $20232 / 8.87$ & 301 & 6 & 26 & gi|132113 & NCBInr & LC-MS/MS \\
\hline 29 & Rubisco small chain 4, chloroplast precursor [Glycine max] & $20232 / 8.87$ & 94 & 10 & 56 & gi|132113 & NCBInr & MALDI-TOF \\
\hline 30 & Rubisco activase precursor [Datisca glomerata] & $41045 / 7.59$ & 74 & 2 & 6 & gi|3687652 & NCBInr & LC-MS/MS \\
\hline 31 & Rubisco activase [Chenopodium quinoa] & $47925 / 6.56$ & 386 & 6 & 18 & gi|21950712 & NCBInr & LC-MS/MS \\
\hline 32 & Rubisco activase, chloroplast precursor [Vigna radiata] & $48042 / 7.57$ & 123 & 13 & 29 & gi|10720249 & NCBInr & MALDI-TOF \\
\hline 33 & Rubisco activase, chloroplast precursor [Vigna radiata] & $48042 / 7.57$ & 73 & 9 & 22 & gi|10720249 & NCBInr & MALDI-TOF \\
\hline 34 & Rubisco activase, chloroplast precursor[Vigna radiata] & $48042 / 7.57$ & 68 & 11 & 32 & gi|10720249 & NCBInr & MALDI-TOF \\
\hline 35 & $\begin{array}{l}\text { Rubisco activase } \beta \text { form precursor [Deschampsia } \\
\text { antarctica] }\end{array}$ & $47371 / 7.57$ & 86 & 2 & 9 & gi|32481063 & NCBInr & LC-MS/MS \\
\hline 36 & Sedoheptulose-1,7-bisphosphatase [Spinacia oleracea] & $42568 / 5.87$ & 88 & 2 & 5 & gi|2529376 & NCBInr & LC-MS/MS \\
\hline 112 & $\begin{array}{l}\text { Gm_ck31838 Soybean induced by Salicylic Acid } \\
\text { (Sedoheptulose-1,7-bisphosphatase) [Glycine max }]\end{array}$ & $23722 / 7.89$ & 271 & 7 & 23 & gi|31464866 & EST_others & LC-MS/MS \\
\hline 37 & Phosphoribulokinase [Pisum sativum $]$ & $39230 / 5.41$ & 106 & 14 & 40 & gi|1885326 & NCBInr & MALDI-TOF \\
\hline 38 & Ribulose-phosphate 3 -epimerase [Spinacia oleracea] & $30632 / 8.23$ & 233 & 2 & 12 & gi $\mid 3264788$ & NCBInr & LC-MS/MS \\
\hline 39 & Transketolase $[$ Spinacia oleracea $]$ & $80744 / 6.2$ & 238 & 3 & 6 & gi|2529342 & NCBInr & LC-MS/MS \\
\hline 40 & Transketolase [Spinacia oleracea $]$ & $80744 / 6.2$ & 259 & 3 & 6 & gi|2529342 & NCBInr & LC-MS/MS \\
\hline \multicolumn{9}{|c|}{ Functional category 2: Energy/glycolysis/glyoxylate cycle/gluconeogensis } \\
\hline 41 & $\begin{array}{l}\text { Glyceraldehyde-3-phosphate dehydrogenase } \\
\text { [Capsicum annuum] }\end{array}$ & $34126 / 6.34$ & 204 & 3 & 11 & gi|18072799 & NCBInr & LC-MS/MS \\
\hline 42 & $\begin{array}{l}\text { Glyceraldehyde-3-phosphate dehydrogenase } \\
\text { [Capsicum annuum] }\end{array}$ & $34126 / 6.34$ & 311 & 6 & 17 & gi|18072799 & NCBInr & LC-MS/MS \\
\hline 43 & $\begin{array}{l}\text { Glyceraldehyde-3-phosphate dehydrogenase } \\
\text { [Pisum sativum }]\end{array}$ & $43597 / 8.8$ & 294 & 4 & 14 & gi|12159 & NCBInr & LC-MS/MS \\
\hline 44 & $\begin{array}{l}\text { Glyceraldehyde-3-phosphate dehydrogenase A-subunit } \\
\text { precursor [Nicotiana tabacum] }\end{array}$ & $42122 / 6.6$ & 364 & 6 & 17 & gi|170237 & NCBInr & LC-MS/MS \\
\hline 45 & Phosphoglycerate kinase [Nicotiana tabacum] & $50317 / 8.48$ & 116 & 10 & 28 & gi|1161600 & $\begin{array}{l}\text { NCBInr } \\
\quad(\text { cont }\end{array}$ & $\begin{array}{l}\text { MALDI-TOF } \\
\text { d on next page) }\end{array}$ \\
\hline
\end{tabular}


Table 1 (continued)

\begin{tabular}{cl}
\hline SID & Protein identification [species] \\
\hline 46 & Phosphoglycerate kinase [Nicotiana tabacum] \\
13 & Phosphoglycerate kinase precursor [Solanum tuberosum] \\
47 & Homologous to plastidic aldolases [Solanum tuberosum] \\
35 & Plastidic aldolases [Solanum tuberosum] \\
48 & Fructose-bisphosphate aldolase, chloroplast precursor \\
& [Spinacia oleracea] \\
49 & Malate dehydrogenase, cytoplasmic [Mesembryanthemum \\
& crystallinum] \\
50 & Malate dehydrogenase 2, glyoxysomal $[$ Brassica napus $]$ \\
51 & Triosephosphate isomerase [Fragaria $\times$ ananassa $]$ \\
52 & Triosephosphate isomerase [Glycine max] \\
53 & Glycolate oxidase, peroxisomal [Spinacia oleracea $]$ \\
54 & Glycolate oxidase [Lens culinaris] \\
55 & Glycolate oxidase [Mesembryanthemum crystallinum $]$
\end{tabular}

\begin{tabular}{|c|c|c|c|c|c|c|}
\hline T. $\mathrm{Mr} / \mathrm{pI}$ & MO & PM & SC $(\%)$ & Acce. no. & Databases & ID Method \\
\hline $50317 / 8.48$ & 104 & 12 & 36 & gi|1161600 & NCBInr & MALDI-TOF \\
\hline $50594 / 7.68$ & 232 & 4 & 9 & gi|3328122 & NCBInr & LC-MS/MS \\
\hline $38632 / 5.89$ & 289 & 5 & 14 & gi| 1781348 & NCBInr & LC-MS/MS \\
\hline $38632 / 5.89$ & 307 & 5 & 18 & gi|1781348 & NCBInr & LC-MS/MS \\
\hline $42727 / 6.85$ & 153 & 2 & 6 & P16096 & SwissProt & LC-MS/MS \\
\hline $35817 / 6.00$ & 66 & 7 & 31 & O24047 & SwissProt & MALDI-TOF \\
\hline $38043 / 8.14$ & 214 & 2 & 9 & gi|4995091 & NCBInr & LC-MS/MS \\
\hline $33505 / 7.64$ & 76 & 11 & 37 & gi|7650502 & NCBInr & MALDI-TOF \\
\hline $27441 / 5.87$ & 108 & 10 & 51 & gi| 48773765 & NCBInr & MALDI-TOF \\
\hline $40317 / 9.16$ & 261 & 6 & 12 & gi| 121530 & NCBInr & LC-MS/MS \\
\hline $40907 / 9.38$ & 277 & 7 & 15 & gi|228403 & NCBInr & LC-MS/MS \\
\hline $40644 / 9.02$ & 71 & 9 & 28 & gi|1773330 & NCBInr & MALDI-TOF \\
\hline
\end{tabular}

Functional category 2: Energy/electron transport

56 ATPase $\beta$ subunit [Oryza sativa (japonica cultivar-group)]

57 ATPase $\beta$ subunit [Crossosoma californicum]

$58 \quad$ ATPase $\beta$ subunit [Platytheca verticellata]

59 ATPase $\alpha$ subunit [Lotus corniculatus var. japonicus]

60 ATPase $\alpha$ subunit [Phaseolus vulgaris]

61 Ferredoxin I precursor [Glycine max]

62 Ferredoxin-like protein [Glycine max]

$\begin{array}{rrrr}45265 / 5.26 & 94 & 12 & 35 \\ 52019 / 5.20 & 194 & 22 & 57 \\ 51184 / 5.07 & 147 & 21 & 50 \\ 55803 / 5.22 & 136 & 13 & 33 \\ 55595 / 6.51 & 90 & 14 & 31 \\ 18448 / 4.84 & 122 & 3 & 16 \\ 22101 / 4.45 & 131 & 2 & 13\end{array}$

Functional category 2: Energy/photosynthesis

63 Chlorophyll $a / b$ binding protein (LHCP AB 180) [Arabidopsis thaliana]

64 PSI PsaN subunit precursor [Zea mays]

65 PSI D2 subunit [Nicotiana sylvestris]

66 PSI reaction centre subunit IV A [Glycine max]

67 PSI reaction centre subunit D precursor [Solanum tuberosum]

68 PSII $10 \mathrm{KD}$ Polypeptide precursor [Glycine max]

69 PSII Oxygen-evolving enhancer protein 1, chloroplast precursor [Pisum sativum]

70 PSII Oxygen-evolving enhancer protein 1, chloroplast precursor [Pisum sativum]

71 PSII Oxygen-evolving enhancer protein 2, chloroplast precursor [Pisum sativum]

72 PSII Oxygen-evolving enhancer protein 2, [Solanum tuberosum]

73 PSII Oxygen-evolving enhancer protein 2 precursor [Glycine max]

74 PSII oxygen-evolving complex protein 3 [Nicotiana tabacum]

75 PSII oxygen-evolving complex protein 3 [Lycopersicon esculentum]

76 Chloroplast Rieske FeS protein [Pisum sativum]

77 Plastocyanin [Cucurbita pepo]

78 Ferredoxin-NADP + reductase [Arabidopsis thaliana]

48 Quinone oxidoreductase-like protein, chloroplast precursor [Arabidopsis thaliana]

79 Carbonic anhydrase [Vigna radiata]

80 Carbonic anhydrase [Vigna radiata]

81 Carbonic anhydrase [Vigna radiata]

Functional category 4: Transcription

82 Chloroplast mRNA-binding protein CSP41 precursor [Glycine max]

119 Putative RNA-binding protein [Arabidopsis thaliana]

Functional category 5: Protein synthesis

83 30S ribosomal protein S5 [Arabidopsis thaliana]

84 50S Ribosomal protein L1, chloroplast precursor [Glycine soja]

\begin{tabular}{|c|c|c|c|c|c|c|}
\hline $25036 / 5.12$ & 103 & 2 & 6 & gi|16374 & NCBInr & LC-MS/MS \\
\hline $12841 / 8.37$ & 75 & 2 & 20 & gi|2981214 & NCBInr & LC-MS/MS \\
\hline $22467 / 9.78$ & 106 & 2 & 13 & gi|19748 & NCBInr & LC-MS/MS \\
\hline $16368 / 9.08$ & 143 & 4 & 13 & gi|5606709 & EST_others & LC-MS/MS \\
\hline $22849 / 9.63$ & 72 & 11 & 44 & gi|34787117 & NCBInr & MALDI-TOF \\
\hline $13753 / 9.73$ & 113 & 2 & 18 & gi|607356 & EST_others & LC-MS/MS \\
\hline $35100 / 6.25$ & 91 & 9 & 27 & gi|20621 & NCB্nr & MALDI-TOF \\
\hline $35100 / 6.25$ & 66 & 7 & 23 & P14226 & SwissProt & MALDI-TOF \\
\hline $28201 / 8.29$ & 169 & 6 & 14 & P16059 & SwissProt & LC-MS/MS \\
\hline $28158 / 8.27$ & 120 & 3 & 8 & gi|1771778 & NCBInr & LC-MS/MS \\
\hline $19825 / 4.81$ & 200 & 6 & 28 & gi|16995778 & EST_others & LC-MS/MS \\
\hline $35377 / 5.89$ & 85 & 9 & 31 & gi|505482 & NCBInr & MALDI-TOF \\
\hline $24557 / 9.64$ & 85 & 3 & 15 & gi|51457944 & NCBInr & LC-MS/MS \\
\hline $24683 / 8.63$ & 190 & 6 & 16 & gi|20832 & NCBInr & LC-MS/MS \\
\hline $10544 / 4.34$ & 63 & 3 & 42 & gi|130265 & NCBInr & LC-MS/MS \\
\hline $40643 / 8.32$ & 381 & 6 & 18 & gi|20465661 & NCBInr & LC-MS/MS \\
\hline $41132 / 8.46$ & 234 & 4 & 8 & Q9ZUC1 & SwissProt & LC-MS/MS \\
\hline $35804 / 7.59$ & 305 & 8 & 20 & gi|8954289 & NCBInr & LC-MS/MS \\
\hline $35804 / 7.59$ & 292 & 8 & 25 & gi|8954289 & NCBInr & LC-MS/MS \\
\hline $35804 / 7.59$ & 267 & 7 & 14 & gi|8954289 & NCBInr & LC-MS/MS \\
\hline $22707 / 5.31$ & 169 & 6 & 21 & gi|7639890 & EST_others & LC-MS/MS \\
\hline $42303 / 7.71$ & 241 & 4 & 12 & gi|3850621 & NCBInr & LC-MS/MS \\
\hline $32682 / 8.99$ & 128 & 2 & 8 & gi|21593322 & NCBInr & LC-MS/MS \\
\hline $29084 / 9.43$ & 119 & 2 & 9 & gi|26046597 & NCBInr & LC-MS/MS \\
\hline
\end{tabular}

MALDI-TOF

MALDI-TOF

MALDI-TOF

MALDI-TOF

MALDI-TOF

gi|169318 NCBInr

gi|5666556 EST_others

gi|23731638 EST_others

LC-MS/MS

LC-MS/MS

$\begin{array}{lll}\text { gi|56784992 } & \text { NCBInr } & \text { MALDI-TOF } \\ \text { gi|14718020 } & \text { NCBInr } & \text { MALDI-TOF } \\ \text { gi|7708546 } & \text { NCBInr } & \text { MALDI-TOF } \\ \text { gi|13358984 } & \text { NCBInr } & \text { MALDI-TOF } \\ \text { gi|169318 } & \text { NCBInr } & \text { MALDI-TOF } \\ \text { gi|5666556 } & \text { EST_others } & \text { LC-MS/MS } \\ \text { gi|23731638 } & \text { EST_others } & \text { LC-MS/MS }\end{array}$


Table 1 (continued)

\begin{tabular}{|c|c|c|c|c|c|c|c|c|}
\hline SID & Protein identification [species] & T. $\mathrm{Mr} / \mathrm{pI}$ & MO & $\mathrm{PM}$ & $\mathrm{SC}(\%)$ & Acce. no. & Databases & ID Method \\
\hline 85 & 50S Ribosomal protein L12 [Glycine $\max ]$ & $16769 / 4.64$ & 467 & 6 & 53 & gi|22927916 & EST_others & LC-MS/MS \\
\hline 86 & Elongation factor-1 (EF-1a) [Glycine max] & $49689 / 9.14$ & 264 & 7 & 17 & gi|18765 & NCBInr & LC-MS/MS \\
\hline 87 & Elongation factor $2(\mathrm{EF}-2)[$ Beta vulgaris $]$ & $94708 / 5.93$ & 56 & 14 & 19 & O23755 & SwissProt & MALDI-TOF \\
\hline 88 & Translation elongation factor-TU [Glycine max $]$ & $52177 / 6.21$ & 170 & 22 & 55 & gi|18776 & NCBInr & MALDI-TOF \\
\hline 89 & Translation elongation factor $\mathrm{G}$ [Glycine $\max ]$ & $77866 / 5.04$ & 465 & 9 & 14 & gi|402753 & NCBInr & LC-MS/MS \\
\hline \multicolumn{9}{|c|}{ Functional category 6: Protein destination and storage } \\
\hline 90 & Stem $28 \mathrm{kDa}$ protein [Glycine $\max ]$ & $29218 / 8.75$ & 74 & 8 & 29 & gi|169898 & NCBInr & MALDI-TOF \\
\hline 91 & Stem $28 \mathrm{kDa}$ protein [Glycine max] & $29218 / 8.75$ & 82 & 9 & 33 & gi|169898 & NCBInr & MALDI-TOF \\
\hline 92 & Stem $28 \mathrm{kDa}$ protein precursor [Glycine $\max ]$ & $29218 / 8.75$ & 61 & 7 & 30 & P15490 & SwissProt & MALDI-TOF \\
\hline 93 & Vegetative storage protein, precursor [ Glycine $\max ]$ & $29433 / 6.72$ & 217 & 4 & 16 & gi|72303 & NCBInr & LC-MS/MS \\
\hline 94 & $\begin{array}{l}\text { PS II stability/assembly factor HCF } 136 \text {, chloroplast } \\
\text { precursor [Arabidopsis thaliana] }\end{array}$ & $44133 / 6.79$ & 61 & 10 & 28 & O82660 & SwissProt & MALDI-TOF \\
\hline 95 & Chaperonin precursor [Pisum sativum] & $63287 / 5.85$ & 105 & 10 & 24 & gi|806808 & NCBInr & MALDI-TOF \\
\hline 96 & Chaperonin precursor $[$ Pisum sativum $]$ & $63287 / 5.85$ & 110 & 10 & 24 & gi|806808 & NCBInr & MALDI-TOF \\
\hline 113 & $\begin{array}{l}\text { Gm_ck } 32525 \text { Soybean induced by SA (chaperonin } \\
\text { 2) }[\text { Glycine } \max ]\end{array}$ & $24022 / 5.44$ & 249 & 4 & 33 & gi|31465298 & EST_others & LC-MS/MS \\
\hline 97 & $\begin{array}{l}\text { Endoplasmic reticulum HSC70-cognate binding } \\
\text { protein precursor [ Glycine max] }\end{array}$ & $73822 / 5.15$ & 88 & 15 & 23 & gi|2642238 & NCBInr & MALDI-TOF \\
\hline 98 & $70 \mathrm{kDa}$ heat shock protein [Phaseolus vulgaris] & $72721 / 5.95$ & 87 & 19 & 27 & gi|22636 & NCBInr & MALDI-TOF \\
\hline 99 & Heat shock protein 70 [Cucumis sativus] & $75480 / 5.15$ & 164 & 16 & 25 & gi|1143427 & NCBInr & MALDI-TOF \\
\hline 100 & Chaperonin groEL $[$ Ricinus communis $]$ & $52461 / 4.77$ & 127 & 12 & 32 & gi|72958 & NCBInr & MALDI-TOF \\
\hline 101 & $\begin{array}{l}\text { Endoplasmic reticulum HSC70-cognate binding } \\
\text { protein precursor [ Glycine } \max ]\end{array}$ & $73822 / 5.15$ & 92 & 14 & 21 & gi|2642238 & NCBInr & MALDI-TOF \\
\hline 76 & $\begin{array}{l}\text { Cyclophilin, chloroplast precursor [Oryza sativa } \\
\text { (japonica cultivar-group)] }\end{array}$ & $25273 / 8.05$ & 123 & 3 & 23 & gi|34902534 & NCBInr & LC-MS/MS \\
\hline 102 & Cyclophilin $[$ Phaseolus vulgaris $]$ & $18376 / 8.36$ & 265 & 7 & 33 & gi|829119 & NCBInr & LC-MS/MS \\
\hline 103 & Cyclophilin [Glycine max] & $18395 / 8.7$ & 174 & 2 & 15 & gi|17981611 & NCBInr & LC-MS/MS \\
\hline 104 & Polyubiquitin 1 [Phaseolus vulgaris] & $6547 / 6.51$ & 67 & 8 & 63 & gi|33327284 & NCBInr & MALDI-TOF \\
\hline 105 & Endopeptidase Clp ATP-binding chain cd4B & $102463 / 5.86$ & 102 & 29 & 30 & gi|9758239 & NCBInr & MALDI-TOF \\
\hline
\end{tabular}

Functional category 7: Transporters

106 Voltage-dependent anion-selective channel

(VDAC1.2) [Lotus corniculatus var. japonicus]

$29696 / 8.57 \quad 93 \quad 2 \quad 8$

$\begin{array}{rrrrlll}28471 / 7.88 & 131 & 3 & 10 & \text { gi|38679315 } & \text { NCBInr } & \text { LC-MS/MS } \\ 35985 / 7.62 & 70 & 11 & 40 & \text { gi|1256608 } & \text { NCBInr } & \text { MALDI-TOF } \\ & & & & & & \\ 22365 / 4.84 & 146 & 3 & 16 & \text { P25096 } & \text { SwissProt } & \text { LC-MS/MS }\end{array}$

$\begin{array}{rrrr}27180 / 5.65 & 154 & 12 & 58 \\ 27180 / 5.65 & 71 & 8 & 4 \\ 57043 / 6.80 & 123 & 15 & 32 \\ 16762 / 4.69 & 282 & 4 & 3 \\ & & & \\ 18108 / 8.20 & 221 & 4 & 25 \\ 14120 / 5.43 & 98 & 2 & 2 \\ 28776 / 5.17 & 232 & 6 & 25\end{array}$

$50808 / 5.83 \quad 87 \quad 12 \quad 28$

gi|35187000 NCBInr

MALDI-TOF

Functional category 20: Secondary metabolism

118 1-Deoxy-D-xylulose 5-phosphate reductoisomerase [Pueraria montana var. lobata]

Functional category 12: Unclear classification

$116 \begin{aligned} & \text { Hypothetical protein [Oryza sativa (japonica cultivar- } \\ & \text { group)] }\end{aligned}$

The data were classified according to protein function described by Bevan et al. (1998) and included an assigned spot number (Fig. 1). SID: Spot ID; T. $\mathrm{Mr} / \mathrm{pI}$ : theoretical molecular weight and $\mathrm{pI}$; MO: MOWSE score; PM: the number of peptides matched; SC: the percentage of sequence coverage; Acce. no.: Accession number; ID method: identification method. The assigned protein of the best matched was given with the species in which it has been identified and its accession number. 
mass maps were obtained. Of the 260 protein spots processed, 53 proteins were successfully identified by querying NCBInr and SwissProt databases using the Mascot search engine. The results are listed in Table 1. Data in Table 1 include an assigned protein spot number, theoretical $\mathrm{pI}$ and $\mathrm{Mr}$, protein identity and its original species, number of peptides matched, percentage sequence coverage, MOWSE score, database searched, identification method and accession number of the best match. Although MALDI-TOF-MS analysis is easy to automate and allows high throughput analysis, protein identification relies solely on the accurate matching of the peptide mass, and it is very difficult to correctly identify proteins if there is a protein mixture. Also the database must contain enough of the protein sequence to compare with the experimentally derived mass map.

LC-MS/MS analysis of 67 spots that were not identified by MALDI-TOF MS allowed the identification of 66 spots, searching against the NCBInr, SwissProt and EST-others databases. The identifications are listed in Table 1. The efficiency of the identification is higher than seen in a proteomic study of rice where $77 \%$ of proteins were identified (Lin et al., 2005). An example of an LC-MS/MS spectrum obtained from spot 4 is presented in Fig. 2. The tandem mass spectrometric method is technically more complex and less scalable than MALDI fingerprinting. Its main advantage is that sequence information derived from several peptides is much more specific for the protein than a list of peptide masses. A short region of local identity, which spans two or more consecutive tryptic cleavage sites may enable identification, even when the remainder of the sequence is divergent (Pandey and Mann, 2000; Newton et al., 2004). In addition, the fragmentation data can be used to search nucleotide databases such as db EST as well as the protein databases. Most of the 119 identified protein spots were identified by searching against the NCBInr database and contained only one protein, seven spots contained two different proteins (spot 6, 13, 35, 48, 63, 76, and 116). Fourteen spots were identified from EST_others database, and 13 spots were identified from the SwissProt database. Only $35 \%$ of the identifications came from G. max or Glycine soja (G. soja) species.

The theoretical and experimental Mrs and pIs matched closely for 72 of the 119 identified spots. We used the BLAST tool in ExPASy (Expert Protein Analysis System) proteomics server of the Swiss Institute of Bioinformatics (http://us.expasy.org/) for annotation and found that many of the proteins identified have a signal peptide. We calculated the theoretical $\mathrm{pI} / \mathrm{Mr}$ of these proteins without the signal sequence using the compute $\mathrm{pI} / \mathrm{Mr}$ tool and found that additional 23 spots have matched Mrs and pIs. For example, the identification for spot 94 is photosystem (PS) II stability/assembly factor HCF136 precursor that has 403 amino acids, and $\mathrm{pI} / \mathrm{Mr}$ of $6.79 / 44133$. The first 78 amino acids is signal peptide, so the theoretical $\mathrm{pI} / \mathrm{Mr}$ of the rest peptide is $5.15 / 35843$, which closely

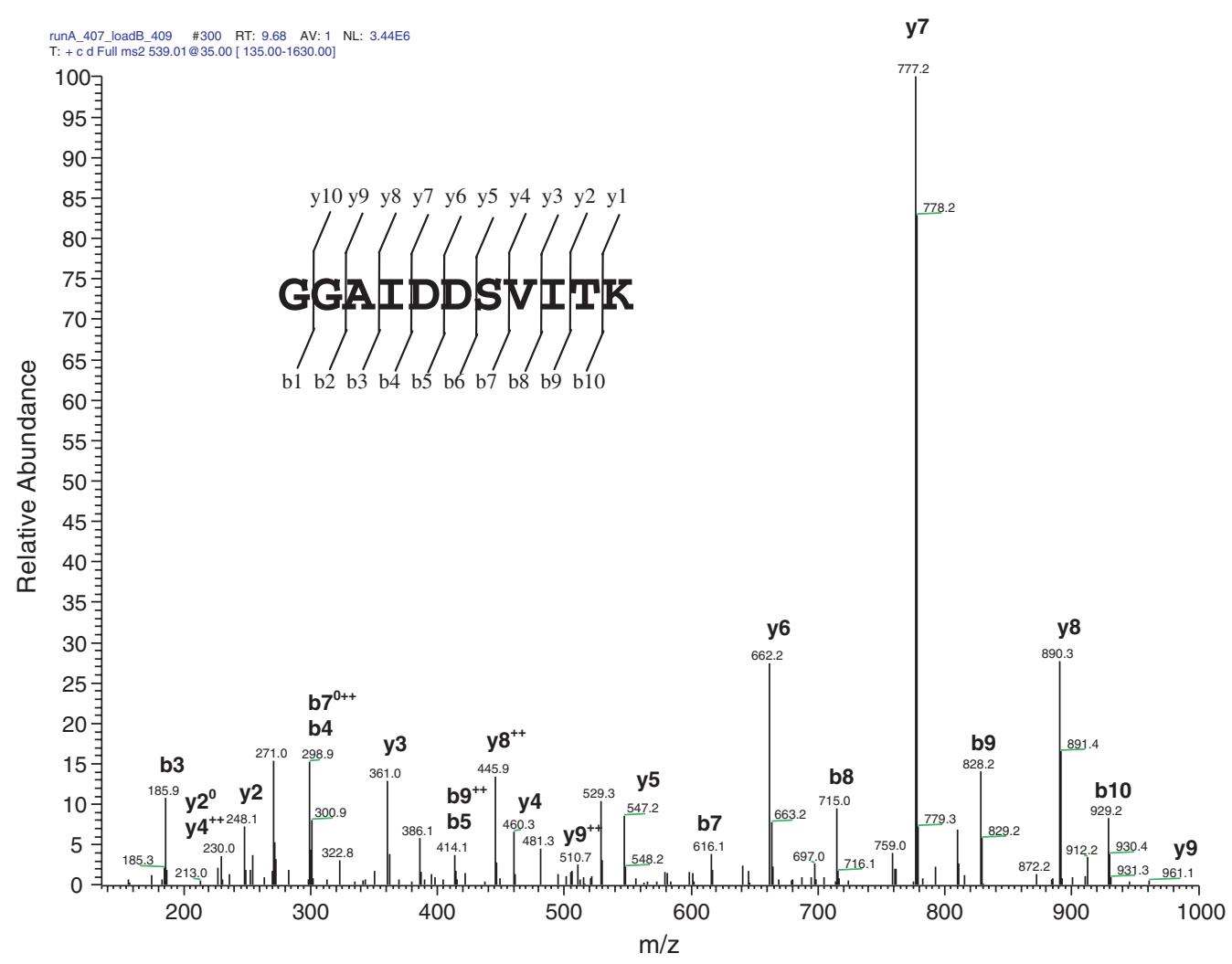

Fig. 2. MS/MS spectrum of one tryptic peptide of spot 4. The protein was identified as T-protein of the glycine cleavage system on the basis of finding 10 unique tryptic peptides, eight with significant ion score, and total protein coverage of $25 \%$. 
matches with our observed values. The $\mathrm{pI} / \mathrm{Mr}$ discrepancy for the other spots might be due to different amino acid sequence from different species (spot 6, 79, 80, 81, 106, and 116), amino acid sequence derived from an EST that may not include complete or accurate sequence (spot 21, 22,82 and 112), or co- and/or post-translational modification (spot 13, 29, and 86).

Multiple spots for a single protein are commonly found on 2D gels (Sarnighausen et al., 2004; Giavalisco et al., 2005). In this study 30 proteins have multiple spots: Tprotein of glycine cleavage system (spot 3-6); Rubisco both large (spot 6, 23, and 24) and small (spot 25-29) subunits; Rubisco activase (spot 30-35); stem $28 \mathrm{kDa}$ protein (spot 90-93); and many others. Several factors may be responsible for this phenomenon. The migration of proteins on a 2D-PAGE gel is very sensitive to small structural differences. These spots might be different isoforms derived from different genes of a multigene family. The complex genome of soybean is expected to contain multiple copies of many genes, and the distinct biophysical properties might be due to amino acid sequence differences in the different isoforms. Alternatively, one gene product may undergo different co- and/or post-translational modifications that affect its $\mathrm{pI}$ or/and $\mathrm{Mr}$. The multiple spots corresponding to one protein could also be a consequence of artificial modification of proteins, such as carbamylation, during the extraction or separation procedure (Berven et al., 2003). However, appropriate precautions were used to prevent artificial modifications and the multiple spots are highly reproducible. Therefore, it is unlikely that the multiple spots in this study are artifacts of protein damage during sample preparation. Taking into account the multiplicity of the spots, we identified a total of 71 unique proteins on our gel.

Based on bioinformatic protein sequence analysis, proteins with at least one transmembrane-spanning domain constitute approximately $20 \%$ of all proteins in eukaryotic genomes (Wallin and von Heijne, 1998; Stevens and Arkin, 2000). These proteins are often underrepresented on 2DPAGE gels due to the tendency of the hydrophobic transmembrane regions to cause the proteins to precipitate, mainly during isoelectric focusing (IEF) (Molloy et al., 1998; Santoni et al., 2000). The observation of plant proteins in 2D-PAGE relative to their general average hydropathicity score has been discussed (Millar et al., 2001). Most proteins identified in this study were expected to be soluble proteins, given that TCA precipitation results in the loss of integral membrane proteins (Wang et al., 2000). Nevertheless, we found some spots that were identified as integral or peripheral membrane proteins, such as chlorophyll $a / b$ binding protein (spot 63), oxygen-evolving enhancer protein of PS II (spot 69-75), and subunits of the PS I complex (spot 64-67). These light-harvesting complexes of PS I and PS II are highly abundant in the thylakoid membranes of plant chloroplasts (Gomez et al., 2000). Therefore, a small fraction of these proteins was extracted during the sample preparation from the whole leaves.

\subsection{Functional distribution of identified proteins}

Identified proteins were classified according to their functions in the categories described by Bevan et al. (1998). In our study, more than 50\% of the identified protein spots are involved in energy metabolism (Fig. 3). This category was divided into four subcategories: the pentose phosphate pathway, the glycolysis pathway/glyoxylate cycle/gluconeogenesis, electron transport, and photosynthesis. The most abundant proteins were also included in the energy category, and most of these proteins appeared as multiple spots. The main function of a plant leaf is energy harvesting, conversion, and storage. Therefore, it is not surprising that a significant number of abundant proteins in the leaf proteome are involved in energy metabolism. The identified proteins involved in photosynthetic electronic transport were: subunits of PS I (spot 64-67) and PS II (spot 63, 68-75); Rieske FeS protein (spot 76); plastocyanin (spot 77); and ferredoxin (spot 61 and 62). Several enzymes responsible for carbon metabolism were also identified: rubisco (spot 6, and 23-29); malate dehydrogenase (spot 49 and 50); sedoheptulose-1,7-biphosphatase (spot 36 and 112); phosphoglycerate kinase (spot 13, 45, and 46); glyceraldehyde-3-phosphate dehydrogenase (spot 41-44); triosephosphate isomerase (spot 51 and 52); and transketolase (spot 39 and 40). Rubisco is the primary enzyme in photosynthetic carbon fixation and the likely rate-limiting factor for photosynthesis under light-saturated conditions and atmospheric $\mathrm{CO}_{2}$ pressure (Makino et al., 1985). The ATPase consists of two parts; a hydrophobic membrane-bound portion called $\mathrm{CF}_{0}$, and a soluble portion that sticks out into the stroma called $\mathrm{CF}_{1} \cdot \mathrm{CF}_{1}$ consists of 5 different subunits: $\alpha-, \beta-, \gamma-, \delta$-, and $\varepsilon$-units (Taiz and Zeiger, 2002). Only the soluble $\alpha-$ (spot 59 and

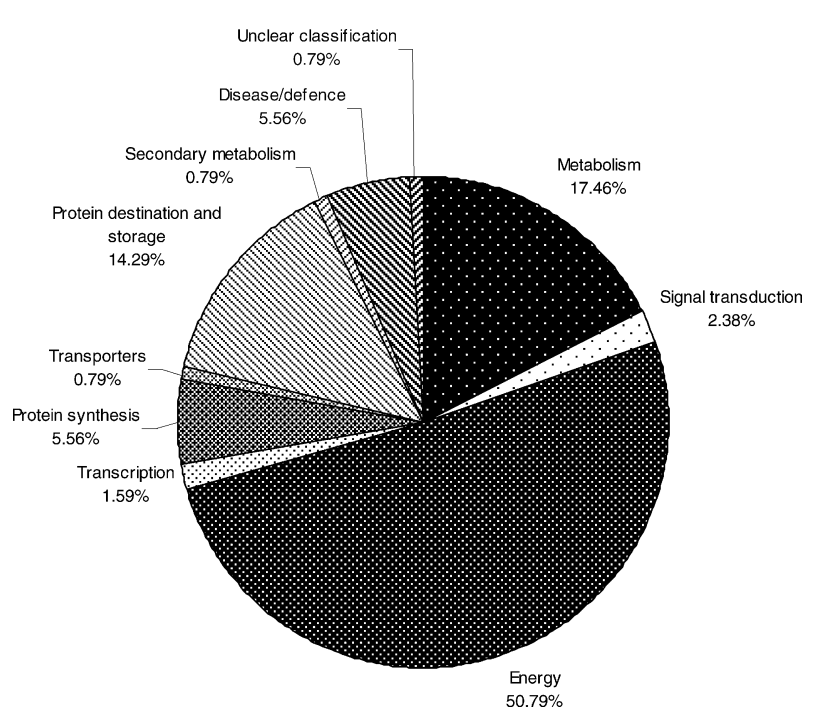

Fig. 3. Assignment of the identified proteins to functional categories using the classification described by Bevan et al. (1998). A total of 119 spots representing 71 different proteins were classified. If a spot contained two proteins it was counted twice. 
60 ) and $\beta$ - (spot 56-58) subunits of the ATPase complex were present on our 2D-PAGE gels.

About $17 \%$ of the identified protein spots are involved in general metabolism. Most of these are involved in amino acid metabolism: glutamine synthetase (spot 11-13); glycine (serine) hydroxymethyltransferase (spot 16-19); alanine aminotransferase (spot 9 and 10); methionine synthase (spot 14); aspartate transaminase ( $\operatorname{spot} 15)$; P(spot 7 and 8), and $\mathrm{H}-$ (spot 1 and 2) and T-protein (spot 3-6) of the glycine cleavage system. Glycine (serine) hydroxymethyltransferase (spot 16-19) catalyzes the interconversion of serine and glycine. It is a key enzyme in the biosynthesis of purines, lipids, hormones and other compounds (Kopriva and Bauwe, 1995). The glycine cleavage system catalyzes the degradation of glycine and is composed of four proteins: $\mathrm{P}, \mathrm{T}, \mathrm{L}$ and $\mathrm{H}$ proteins (Bourguignon et al., 1993). Other identified proteins belong in the protein destination and storage category: chaperonin (spot 95, 96, 100 and 113), HSP/HSC (heat shock protein/heat shock cognate) 70 and associated co-chaperones (spot 97, 98, 99, and 101), stem $28 \mathrm{kDa}$ protein (spot 90-93), cyclophilin (spot 76, 102, and 103), endopeptidase Clp (spot 105) and polyubiquitin (spot 104). HSPs are associated with protein folding, protein translocation across membranes, assembly of oligomeric proteins, modulation of receptor activities, mRNA protection, prevention of enzyme denaturation and their stress-induced aggregation, and with post-stress ubiquitin and chaperonin-aided repair. Based on these functions, HSPs have been termed "molecular chaperones" (Georgopoulos and Welch, 1993; Leone et al., 2000). Stem $28 \mathrm{kDa}$ glycoprotein is also known as vegetative storage protein $\mathrm{A}$. It may function as a somatic storage protein during early seedling development and mainly accumulates in the stem of developing seedlings (Mason et al., 1988).

Additional identified protein spots are included in disease/defence category: ascorbate peroxidase (spot 109 and 110); catalase (spot 111); stress-induced protein SAM22 (spot 114); peroxiredoxin (spot 116 and 117); and PR1 (spot 115). Three signal transduction proteins were detected in our study: harpin-binding protein 1 (spot 63); P21 protein (spot 108); and $\mathrm{G}$ protein (spot 107). Other identified proteins are involved in protein synthesis (ribosomal protein, spot 83-85; elongation factor, spot 86-89), in transcription (RNA binding protein, spot 119 and 82), in ion transport (voltage-dependent anion-selective channel, spot 106), and in secondary metabolism (1-deoxy-D-xylulose 5-phosphate reductoisomerase, spot 118). Only one identified protein has unknown function (spot 116). None of the identified proteins are in the cell growth/division, intracellular traffic, cell structure or transposon categories.

\section{Conclusion}

We separated soybean leaf proteins using 2D-PAGE and identified 119 protein spots with MALDI-TOF MS and LC-MS/MS. The broad dynamic range of protein expression is one of the major difficulties in separation of soybean leaf proteins by 2D-PAGE. LC-MS/MS is a more powerful and sensitive way to obtain positive identifications from 2D-PAGE spots, although it is more laborious and more difficult to automate than MALDI-TOF-MS analysis. Future studies of leaf physiology will benefit from this proteome reference map of soybean leaf.

\section{Experimental}

\subsection{Plant material}

The seeds of soybean cultivar Clark were grown on horticultural vermiculite in 1-L pots in the greenhouse at the University of Maryland, College Park, MD. Plants were transferred to the field site at the USDA, Beltsville Southfarm just before the cotyledons emerged, and grown under full sunlight. Plants were watered and rotated daily, and fertilized with Hoagland solution every 3 days. Primary leaves were harvested when they were 12 days old. Four biological replicate samples were used for protein extraction and 2D-PAGE analysis. Samples were immediately frozen in liquid nitrogen, and stored at $-80{ }^{\circ} \mathrm{C}$ prior to analysis.

\subsection{Protein extraction from leaves}

Frozen sample was ground in a mortar with liquid nitrogen and incubated with 10\% TCA and $0.07 \%$ 2-mercaptoethanol in acetone for $1 \mathrm{~h}$ at $-20{ }^{\circ} \mathrm{C}$ (Natarajan et al., 2005). The precipitated proteins were pelleted and washed with ice-cold acetone containing $0.07 \%$ 2-mercaptoethanol to remove pigments and lipids until the supernatant was colorless. The pellet was vacuum dried, resuspended in resolubilization solution (9 M urea, 1\% CHAPS, $1 \%$ DTT, $1 \%$ pharmalyte) and sonicated to extract proteins. Insoluble tissue was removed by centrifugation at $21,000 \mathrm{~g}$ for $30 \mathrm{~min}$. Protein concentration was determined according to Bradford (1976) using a commercial dye reagent (Bio-Rad Laboratories, Hercules, CA) with bovine serum albumin as a standard.

\section{3. $2 D-P A G E$}

An IPGPhor apparatus (GE Healthcare, Piscataway, $\mathrm{NJ})$ was used for IEF with immobilized $\mathrm{pH}$ gradient (IPG) strips $(\mathrm{pH} 3.0-10.0$, linear gradient, $13 \mathrm{~cm})$. The IPG strips were rehydrated $12 \mathrm{~h}$ with $250 \mu \mathrm{L}$ rehydration buffer ( $8 \mathrm{M}$ urea, $2 \%$ CHAPS, $0.5 \%$ pharmalyte, $0.002 \%$ bromophenol blue) containing $350 \mu \mathrm{g}$ proteins. The voltage settings for IEF was $500 \mathrm{~V}$ for $1 \mathrm{~h}, 1000 \mathrm{~V}$ for $1 \mathrm{~h}, 5000 \mathrm{~V}$ for $1 \mathrm{~h}$, and $8000 \mathrm{~V}$ to a total $46.86 \mathrm{kVh}$. Following electrophoresis, the protein in the strips was denatured with equilibration buffer ( $50 \mathrm{mM}$ Tris- $\mathrm{HCl} \mathrm{pH} 8.8,6 \mathrm{M}$ urea, $30 \%$ glycerol, $2 \%$ SDS, $0.002 \%$ bromophenol blue, $1 \%$ DTT) 
and then incubated with the same buffer containing $2.5 \%$ iodoacetamide instead of DTT for $30 \mathrm{~min}$ at room temperature. The second dimension electrophoresis was performed on a $12.5 \%$ gel using a Hoefer SE 600 Ruby electrophoresis unit (GE Healthcare, Piscataway, NJ). The gels were stained with CBB G-250 (Newsholme et al., 2000) and scanned using a Personal Densitometer SI (GE Healthcare, Piscataway, NJ).

\subsection{In-gel digestion of protein spots}

Protein digestion was performed as described previously (Natarajan et al., 2005). Spots were excised from the stained gel and washed with $\mathrm{CH}_{3} \mathrm{CN}: \mathrm{H}_{2} \mathrm{O}(1: 1, \mathrm{v} / \mathrm{v})$ containing $25 \mathrm{mM}$ ammonium bicarbonate to remove the dye. The gel plug was dehydrated with $100 \%$ acetonitrile, and was dried under vacuum and incubated overnight at $37{ }^{\circ} \mathrm{C}$ with $20 \mu \mathrm{L}$ of $10 \mu \mathrm{g} / \mathrm{mL}$ porcine trypsin in $20 \mathrm{mM}$ ammonium bicarbonate. The resulting tryptic fragments were eluted by diffusion into $\mathrm{CH}_{3} \mathrm{CN}: \mathrm{H}_{2} \mathrm{O}(1: 1, \mathrm{v} / \mathrm{v})$ and $0.5 \%$ trifluoroacetic acid. A sonic bath was used to facilitate the diffusion. The extract was vacuum dried and the pellet was dissolved in $\mathrm{CH}_{3} \mathrm{CN}: \mathrm{H}_{2} \mathrm{O}(1: 1, \mathrm{v} / \mathrm{v})$ and $0.1 \%$ trifluoroacetic acid.

\subsection{Mass spectrometry}

For PMF a Voyager DE-STR MALDI-TOF mass spectrometer (Applied Biosystems, Framingham, MA) operated in positive ion reflector mode was used to analyze tryptic peptides. Samples were co-crystallized with $\alpha$-cyanohydroxycinnamic acid (CHCA) matrix, and spectra were acquired with 50 shots of a $337 \mathrm{~nm}$ Nitrogen Laser operating at $20 \mathrm{~Hz}$. Spectra were calibrated using the trypsin autolysis peaks at $m / z 842.51$ and 2.211.10 as internal standards. For MS/MS a Thermo Finnigan LCQ Deca XP plus Ion Trap mass spectrometer was used to analyze proteins that were not positively identified by MALDI-TOF-MS. Peptides were separated on a reverse phase column using a $30 \mathrm{~min}$ gradient of $5-60 \%$ acetonitrile in water with $0.1 \%$ formic acid. The instrument was operated with a duty cycle that acquired MS/MS spectra on the three most abundant ions identified by a survey scan from 300 to $2000 \mathrm{Da}$. Dynamic exclusion was employed to prevent the continuous analysis of the same ions. Once two MS/ MS spectra of any given ion had been acquired, the parent mass was placed on an exclusion list for the duration of $1.5 \mathrm{~min}$. The raw data were processed by Sequest to generate DTA files for database searching. The merge.pl script from Matrix Science was used to convert multiple Sequest DTA files into a single mascot generic file suitable for searching in Mascot.

\subsection{Data analysis}

Protein identification was performed using the Mascot search engine (http://www.matrixscience.com), which uses a probability based scoring system (Perkins et al., 1999). NCBI non-redundant and SwissProt databases were selected as the primary databases to be searched. For LC-MS/MS, if the primary databases did not yield identity, the "EST_others" database was queried. The parameters for database searches with MALDI-TOF PMF data and with MS/MS spectra were set as before (Natarajan et al., 2005). For MALDI-TOF-MS data to qualify as a positive identification, a protein's score had to equal or exceed the minimum significant score of 64 for NCBInr or 55 for SwissProt database searching. Positive identifications of proteins by MS/MS analysis required a minimum of two unique peptides, with at least one peptide having a significant ion score.

\section{Acknowledgements}

The authors thank Dr. W. Kenworthy for providing soybean seeds and Dr. J. Slovin and Dr. N.C. Talbot for their critical reviews of this manuscript. Funding for this research was provided by ARS Project 1275-2100015600D.

\section{References}

Bardel, J., Louwagie, M., Jaquinod, M., Jourdain, A., Luche, S., Rabilloud, T., Macherel, D., Garin, J., Bourguignon, J., 2002. A survey of the plant mitochondrial proteome in relation to development. Proteomics 2, 880-898.

Berven, F.S., Karlsen, O.A., Murrell, J.C., Jensen, H.B., 2003. Multiple polypeptide forms observed in two-dimensional gels of Methylococcus capsulatus (Bath) polypeptides are generated during the separation procedure. Electrophoresis 24, 757-761.

Bevan, M., Bancroft, I., Bent, E., Love, K., Goodman, H., Dean, C., Bergkamp, R., Dirske, W., Van Staveren, M., Stiekema, W., 1998. Analysis of $1.9 \mathrm{Mb}$ of contiguous sequence from chromosome 4 of Arabidopsis thaliana. Nature 391, 485-488.

Bourguignon, J., Vauclare, P., Merand, V., Forest, E., Neuburger, M., Douce, R., 1993. Glycine decarboxylase complex from higher plants. Molecular cloning, tissue distribution and mass spectrometry analyses of the T protein. Eur. J. Biochem. 217, 377-386.

Bradford, M.M., 1976. A rapid and sensitive method for the quantitation of microgram quantities of protein utilizing the principle of proteindye binding. Anal. Biochem. 72, 248-254.

Chang, W., Huang, L., Shen, M., Webster, C., Burlingame, A.L., Roberts, J., 2000. Patterns of protein synthesis and tolerance of anoxia in root tips of maize seedlings acclimated to a low-oxygen environment and identification of proteins by mass spectrometry. Plant Physiol. 122, 295-317.

Ferro, M., Salvi, D., Brugiere, S., Miras, S., Kowalski, S., Louwagie, M., Garin, J., Joyard, J., Rolland, N., 2003. Proteomics of the chloroplast envelope membranes from Arabidopsis thaliana. Mol. Cell. Proteomics $2,325-345$

Gallardo, K., Job, C., Groot, S.P.C., Puype, M., Demol, H., Vanderkerckhove, J., Job, D., 2001. Proteomic analysis of Arabidopsis seed germination and priming. Plant Physiol. 126, 835-848.

Georgopoulos, C., Welch, W.J., 1993. Role of the major heat shock proteins as molecular chaperones. Annu. Rev. Cell Biol. 9, 601-634.

Giavalisco, P., Nordhoff, E., Kreitler, T., Kloppel, K., Lehrach, H., Klose, J., Gobom, J., 2005. Proteome analysis of Arabidopsis thaliana by 
two-dimensional gel electrophoresis and matrix-assisted laser desorption/ionisation-time of flight mass spectrometry. Proteomics 5, 19021913.

Gomez, S., Chitins, P.R., Yunus, M., Pathre, U., Mohanty, P., 2000Probing Photosynthesis: Mechanisms, Regulation, and Adaptation. Taylor \& Francis, London, pp. 51-69.

Komatsu, S., Kojima, K., Suzuki, K., Ozaki, K., Higo, K., 2004. Rice proteome database based on two-dimensional polyacrylamide gel electrophoresis: its status in 2003. Nucl. Acids Res. 32, D388-D392.

Kopriva, S., Bauwe, H., 1995. Serine hydroxymethyltransferase from Solanum tuberosum. Plant Physiol. 107, 271-272.

Kruft, V., Eubel, H., Jansch, L., Werhahn, W., Braun, H.-P., 2001. Proteomic approach to identify novel mitochondrial proteins in Arabidopsis. Plant Physiol. 127, 1694-1710.

Leone, A., Piro, G., Leucci, M.R., Zacheo, G., Dalessandro, G., 2000. Membrane-cell wall-associated heat shock proteins in two genotypes of barley seedlings. Plant Biosyst. 134, 171-178.

Lin, S., Chang, M., Tsai, Y., Lur, H., 2005. Proteomic analysis of the expression of proteins related to rice quality during caryopsis development and the effect of high temperature on expression. Proteomics 5, 2140-2156.

Lonosky, P., Zhang, X., Honavar, V., Dobbs, D., Fu, A., Rodermel, S., 2004. A proteomic analysis of maize chloroplast biogenesis. Plant Physiol. 134, 560-574.

Makino, A., Mae, T., Ohira, K., 1985. Photosynthesis and ribulose-1,5bisphosphate carboxylase/oxygenase in rice leaves from emergence through senescence. Planta 166, 414-420.

Mason, H.S., Guerrero, F.D., Boyer, J.S., Mullet, J.E., 1988. Proteins homologous to leaf glycoproteins are abundant in stems of darkgrown soybean seedlings. Analysis of proteins and cDNAs. Plant Mol. Biol. 11, 845-856.

Mathesius, U., Keijzers, G., Natera, S.H.A., Winman, J.J., Djordjevic, M.A., Rolfe, B.G., 2001. Establishment of a root proteome reference map for the model legume Medicago truncatula using the expressed sequence tag database for peptide mass fingerprinting. Proteomics 1, $1424-1440$.

Millar, A.H., Sweetlove, L.J., Giege, P., Leaver, C., 2001. Analysis of the Arabidopsis mitochondrial proteome. Plant Physiol. 127, 1711-1727.

Molloy, M.P., Herbert, B.R., Walsh, B.J., Tyler, M.I., Sanchez, J.C., Hochstrasser, D.F., Williams, K.L., Gooley, A.A., 1998. Extraction of membrane proteins by differential solubilization for separation using two-dimensional gel electrophoresis. Electrophoresis 19, 837-844.

Mooney, B.P., Thelen, J.J., 2004. High-throughput peptide mass fingerprinting of soybean seed proteins: automated workflow and utility of UniGene expressed sequence tag databases for protein identification. Phytochemistry 65, 1733-1744.

Natarajan, S., Xu, C., Caperna, T.J., Garrett, W.M., 2005. Comparison of protein solubilization methods suitable for proteomic analysis of soybean seed proteins. Anal. Biochem. 342, 214-220.

Newsholme, S.J., Maleeft, B.F., Steiner, S., Anderson, N.L., Schwartz, L.W., 2000. Two-dimensional electrophoresis of liver proteins: char- acterization of a drug-induced hepatomegaly in rats. Electrophoresis 21, 2122-2128.

Newton, R.P., Brenton, A.G., Smith, C.J., Dudley, E., 2004. Plant proteome analysis by mass spectrometry: principles, problems, pitfalls and recent developments. Phytochemistry 65, 1449-1485.

Pandey, A., Mann, M., 2000. Proteomics to study genes and genomes. Nature 405, 837-846.

Perkins, D.N., Pappinl, D.J.C., Creasy, D.M., Cottrell, J.S., 1999. Probability-based protein identification by searching sequence databases using mass spectrometry data. Electrophoresis 20, 35513567.

Porubleva, L., Velden, K.V., Kothari, S., Oliver, D.J., Chitnis, P.R., 2001. The proteome of maize leaves: use of gene sequences and expressed sequence tag data for identification of proteins with peptide mass fingerprints. Electrophoresis 22, 1724-1738.

Santoni, V., Molloy, M., Rabilloud, T., 2000. Membrane proteins and proteomics: un amour impossible? Electrophoresis 21, 1054-1070.

Sarnighausen, E., Wurtz, V., Heintz, D., Dorsselaer, A.V., Resk, R., 2004. Mapping of the Physcomitrella patens proteome. Phytochemistry 65, 1589-1607.

Schiltz, S., Gallardo, K., Huart, M., Negroni, L., Sommerer, N., Burstin, J., 2004. Proteome reference maps of vegetative tissues in pea. An investigation of nitrogen mobilization from leaves during seed filling. Plant Physiol. 135, 2241-2260.

Stevens, T.J., Arkin, I.T., 2000. Do more complex organisms have a greater proportion of membrane proteins in their genomes? Proteins 39, 417-420.

Taiz, L., Zeiger, E., 2002. Photosynthesis: the light reactions. In: Plant physiology. Sinauer Associates, pp. 134-135 (Chapter 7).

Wallin, E., von Heijne, G., 1998. Genome-wide analysis of integral membrane proteins from eubacterial, archaean, and eukaryotic organisms. Protein Sci. 7, 1029-1038.

Wang, Y., Sun, J., Chitnis, P.R., 2000. Proteomic study of the peripheral proteins from thylakoid membranes of the cyanobacterium Synechocystis sp. PCC 6803. Electrophoresis 21, 1746-1754.

Watson, B.S., Asirvatham, V.S., Wang, L., Sumner, L.W., 2003. Mapping the proteome of barrel medic (Medicago truncatula). Plant Physiol. 131, 1104-1123.

Wilson, K.A., McManus, M.T., Gordon, M.E., Jordan, T.W., 2002. The proteomics of senescence in leaves of white clover, Trifolium repens (L.). Proteomics 2, 1114-1122.

Yamaguchi, K., von Knoblauch, K., Subramanian, A.R., 2000. The plastid ribosomal proteins: identification of all the proteins in the $30 \mathrm{~S}$ subunit of an organelle ribosome (chloroplast). J. Biol. Chem. 275, 28455-28465.

Yamaguchi, K., Prieto, S., Beligni, M., Haynes, P.A., McDonald, W.H., Yates, J.R., Mayfield, S., 2002. Proteomic characterization of the small subunit of Chlamydomonas reinhardtii chloroplast ribosome: identification of a novel S1 domain-containing protein and unusually large orthologs of bacterial S2, S3, and S5. Plant Cell 14, 2957-2976. 